Article

\title{
Algebraic Properties of Arbitrage: An Application to Additivity of Discount Functions
}

\author{
Salvador Cruz Rambaud ${ }^{+}$(1) \\ Departamento de Economía y Empresa, Universidad de Almería (Spain), 04120 Almería, Spain; scruz@ual.es \\ + La Cañada de San Urbano, s/n (04120) Almería, Spain.
}

Received: 15 August 2019; Accepted: 16 September 2019; Published: 19 September 2019

check for updates

\begin{abstract}
Background: This paper aims to characterize the absence of arbitrage in the context of the Arbitrage Theory proposed by Kreps (1981) and Clark (2000) which involves a certain number of well-known financial markets. More specifically, the framework of this model is a linear (topological) space $X$ in which a (convex) cone $C$ defines a vector ordering. There exist markets for only some of the contingent claims of $X$ which assign a price $p_{i}$ to the marketed claim $m_{i}$. The main purpose of this paper is to provide some novel algebraic characterizations of the no arbitrage condition and specifically to derive the decomposability of discount functions with this approach. Methods: Traditionally, this topic has been focused from a topological or probabilistic point of view. However, in this manuscript the treatment of this topic has been by using purely algebraic tools. Results: We have characterized the absence of arbitrage by only using algebraic concepts, properties and structures. Thus, we have divided these characterizations into those concerning the preference relation and those involving the cone. Conclusion: This paper has provided some novel algebraic properties of the absence of arbitrage by assuming the most general setting. The additivity of discount functions has been derived as a particular case of the general theory.
\end{abstract}

Keywords: arbitrage; contingent claim; free lunch; discount function; linear price; linear space; preference relation; cone

JEL Classification: G00; G13

\section{Introduction}

Despite its original simplicity, the concept of arbitrage opportunity has been treated in different contexts. For a general revision of the related concepts and the main contributions to this topic, see [1]. One of these approaches (simple arbitrage opportunity) requires zero wealth at time 0 , never loses any wealth and, with strictly positive probability, generates positive wealth at time $T$. The framework is a probabilistic space $(\Omega, \mathcal{F}, P)$, where $\mathcal{F}$ denotes the family of subsets of $\Omega$, called the events, and $P$ is a measure of probability which assigns to each event $B$ in $\mathcal{F}$ its probability $P(B)$. There exist $T+1$ dates: $0,1, \ldots, T$. At each $t \in\{0,1, \ldots, T\}$, a family $\mathcal{F}_{t}$ denotes the set of events corresponding to the available information at instant $t$. That is to say, if an event $B$ is in $\mathcal{F}_{t}$, then at instant $t$ it is well known if this event is true or false. We adopt the usual convention that $\mathcal{F}_{t} \subseteq \mathcal{F}_{s}$ provided that $t \leq s$, which means that the events never "forget". By simplicity, we have also that every event of $\mathcal{F}_{0}$ has probability 0 or 1 , which essentially means that there is not any information at instant $t=0$. Also considered, the filtration $\mathcal{F}=\left\{\mathcal{F}_{0}, \mathcal{F}_{1}, \ldots, \mathcal{F}_{T}\right\}$ represents how the information is revealed through time. An adapted process is a sequence $X=\left\{X_{0}, X_{1}, \ldots, X_{T}\right\}$ such that, for every $t, X_{t}$ is a random variable with respect to $\left(\Omega, \mathcal{F}_{t}\right)$. Informally, this means that, at instant $t$, the result of $X_{t}$ is known [2].

The original model proposed by Clark [3,4] only considered a very clear and intuitive definition of arbitrage (simple free lunch) inside a very general setting (a marketed vector space where a claim is 
allowed to have more than one price) without involving probability. For this reason, the main purpose of this paper is the algebraic treatment of no arbitrage (NA) to fill the gap between the topological and probabilistic approaches recently arisen on this topic (see Section 6). Indeed, this is the significance of this paper.

Thus, the methodology employed in this paper includes a general vector space with an ordering, that is to say, a binary relation satisfying only reflexivity. In the process of searching logical implications, the price correspondence is converted into a map, and a new ordering on the quotient space provides necessary and sufficient conditions for NA. On the other hand, the cone generated by the initial ordering (which represents the "direction of increasing economic value") gives a novel algebraic perspective and several characterizations.

As a consequence, the findings of this paper represent a novelty in the financial theory because, as indicated, they involve purely algebraic tools and structures. These is the main difference between the findings of this manuscript and those reported in the existing literature. Moreover, to the extent of our knowledge, this would be the first essay in characterizing NA with only algebraic tools apart from the work by [5].

The organization of this paper is as follows. After this introduction, Section 2 sets the framework of this paper by proposing the broadest alternatives in order to reach a setting as general as possible. Section 3 clarifies the number of images of the elements in $M$, and provides some necessary and sufficient conditions for NA, involving some requirements on the price map and the preference relation. Section 4 includes some properties of the cones involved in NA and a theorem of characterization of NA by using the quotient space induced by the price map. In Section 5 the additivity of discount functions is deduced with the tools used in this approach. Section 6 discusses the main findings of this paper putting them in relation with the referential contributions about the topic on NA. Finally, Section 7 summarizes and concludes.

Taking into account that the mathematical analysis of this paper (Sections 2-4) is quite extensive and informative, the main concepts and results employed in order to derive an articulate mathematical analysis have been summed up in Figure 1.

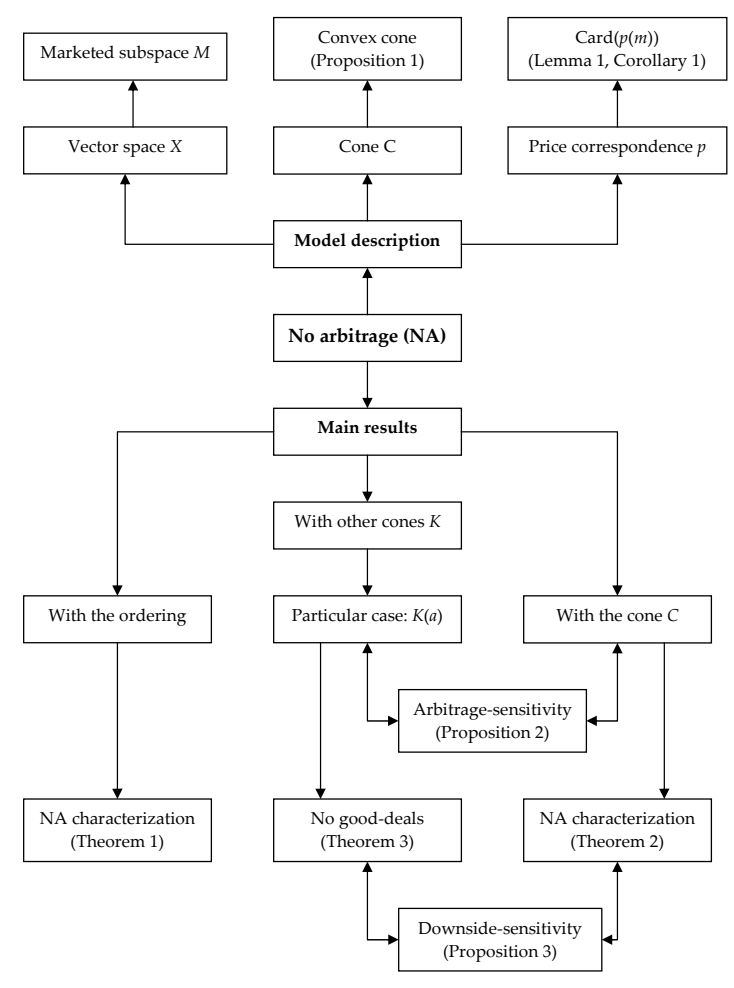

Figure 1. Structure of Sections 2-4. Source: Own elaboration. 


\section{The Model}

The following model, introduced by [3,4], involves a certain number of well-known financial markets, and it was originally proposed at this level of abstraction by [6,7]. First, we are going to describe the framework of this model.

1. $X$ is a linear space with vector ordering $\succeq$ (not necessarily an ordered vector space), which represents the collection of all contingent claims. A contingent claim is a derivative whose future payoffs depend on the value of another underlying asset or, more generally, which is dependent on the realization of some uncertain future events. $X$ can be also interpreted as the payoffs of the claims traded by an agent in a competitive market [8]. The payoff space $X$ is a subset of the contingent claim space $\mathbb{R}^{k}$. In complete markets, we have $X=\mathbb{R}^{k}$, otherwise $X \subset \mathbb{R}^{k}$ [9]. In effect, the initial prices of $m$ risky securities, denoted by $S_{1}(0), \ldots, S_{m}(0)$, are known at time $t=0$. Their values at time $t=1$ are uncertain and depending on $k$ events $\Omega=\left\{\omega_{1}, \omega_{2}, \ldots, \omega_{k}\right\}$ (probabilistic or not: states of the world). The possible values of the asset process at time $t=1$ are listed in the following $k \times m$ matrix:

$$
S(1, \Omega):=\left(\begin{array}{cccc}
S_{1}\left(1 ; \omega_{1}\right) & S_{2}\left(1 ; \omega_{1}\right) & \cdots & S_{m}\left(1 ; \omega_{1}\right) \\
S_{1}\left(1 ; \omega_{2}\right) & S_{2}\left(1 ; \omega_{2}\right) & \cdots & S_{m}\left(1 ; \omega_{2}\right) \\
\vdots & \vdots & \ddots & \vdots \\
S_{1}\left(1 ; \omega_{k}\right) & S_{2}\left(1 ; \omega_{k}\right) & \cdots & S_{m}\left(1 ; \omega_{k}\right)
\end{array}\right)
$$

Usually, in order to analyze the topological properties of arbitrage, $X$ is assumed to be a locally convex, Hausdorff, topological vector space [10] over the real number field, called the consumption space [11]. However, in this paper, we will propose a very general setting.

Also, it can be said that $X$ is a locally convex and Hausdorff topological vector lattice. By definition, a topological vector lattice $X$ is a topological vector space endowed with a continuous operation $\vee: X \times X \rightarrow X$. In effect, the lattice operation $\vee$ generates a natural vector order $\succeq$ on $X$ defined by the following condition:

$$
x \succeq y \text { if, and only if, }(x-y) \vee 0=x-y .
$$

2. $C$ is a (convex) cone, that is to say, a (convex: closed for the sum) subset $C$ such that $x \in C$ implies $\lambda x \in C$, for every positive real number $\lambda$. A strictly positive cone is a cone $C$ such that $0 \notin C$. Observe that the assumption that $0 \notin C$ implies that $C \cup\{0\}$ is a pointed cone, which generates the vector ordering $\succeq$ in $X$ defined by:

$$
x \succeq y \text { if, and only if, } x-y \in C \cup\{0\} .
$$

There exist also some other alternative approaches as, for example, that $C$ is constructed starting from the function of utility of an agent [12].

The vector ordering $\succeq$ :

- Is reflexive. In effect, for every $x \in X, x-x=0 \in C \cup\{0\}$.

- May be antisymmetric. In effect, $x_{1}-x_{2} \in C \cup\{0\}$ and $x_{2}-x_{1} \in C \cup\{0\}$ imply $x_{1}=x_{2}$ if, and only if, either $C \cap(-C)=\varnothing$ or $C$ is a salient cone, i.e., the largest linear subspace contained in $C \cup\{0\}$ is $\{0\}$.

- May be transitive. Observe that $\succeq$ is transitive if, and only if, $C \cup\{0\}$ is convex. In effect, assume that $\succeq$ is transitive. Given $c_{1}$ and $c_{2}$ belonging to $C \cup\{0\}$, one has:

$$
\left(c_{1}+c_{2}\right)-c_{1}=c_{2} \in C \cup\{0\},
$$


whereby

$$
c_{1}+c_{2} \succeq c_{1} \succeq 0 .
$$

By the transitivity of $\succeq, c_{1}+c_{2} \succeq 0$, whereby $c_{1}+c_{2} \in C \cup\{0\}$. Reciprocally, assume that $C \cup\{0\}$ is convex. If $c_{1} \succeq c_{2}$ and $c_{2} \succeq c_{3}$, one has $c_{1}-c_{2} \in C \cup\{0\}$ and $c_{2}-c_{3} \in C \cup\{0\}$. As $C \cup\{0\}$ is convex, $c_{1}-c_{3} \in C \cup\{0\}$ and, consequently, $c_{1} \succeq c_{3}$.

- Satisfies that, for every $x_{1}, x_{2}$ and $x_{3} \in X$, and $0 \leq \lambda \in \mathbb{R}$, the following two properties hold:

- $\quad x_{1} \succeq x_{2}$ if, and only if, $x_{1}+x_{3} \succeq x_{2}+x_{3}$.

- $\quad x_{1} \succeq x_{2}$ if, and only if, $\lambda x_{1} \succeq \lambda \bar{x}_{2}$.

Denote by $\succ$ the asymmetric component of $\succeq$. So:

$$
x_{1} \succ x_{2} \text { if and only if } x_{1} \succeq x_{2} \text { and } x_{1} \neq x_{2} .
$$

Thus,

(a) The positive cone is given by $C \cup\{0\}=\{x \in X: x \succeq 0\}$.

(b) The strictly positive cone is given by $C=\{x \in X: x \succ 0\}$. The role of the strictly positive cone is to offer a direction of increase of the economic value of contingent claims.

(c) In this paper, $C$ is assumed to be a (linear) cone, i.e., in general, $\succeq$ satisfies neither antisymmetry nor transitivity.

Table 1 summarizes the possible properties to be satisfied by $\succeq$ according to the type of cone.

Table 1. Possible properties of $\succeq$ and types of cones. Source: Own elaboration.

\begin{tabular}{cc}
\hline Property of $\succeq$ & Type of Cone \\
\hline Reflexivity & Linear \\
Antisymmetry & Salient \\
Transitivity & Convex \\
Compatibility with the sum & Linear \\
Compatibility with the product by a positive scalar & Linear \\
\hline
\end{tabular}

3. There exist markets for only some of the contingent claims of $X$, say $\left\{m_{i}\right\}_{i \in I}$, and the collection of the marketed claims $M$, called the marketed claims space, is the linear subspace spanned by $\left\{m_{i}\right\}_{i \in I}$. Thus, a marketed claim $m \in M$ is a portfolio of short and long positions (an investor has long positions if such investor has bought and owns those shares of stocks, whilst the investor has short positions of $\mathrm{s} /$ he owes those stocks to someone) in the available markets. We will employ the notation $m=\sum_{i \in I} \lambda_{i} m_{i}$ for a marketed claim where the number of proportions $\lambda_{i}$ of the claim $m_{i}$ is non null only for a finite number of markets. Therefore, the number of markets is not necessarily finite [5]. In short, we have the following equality:

$$
M=\left\{x \in X: x=\sum_{i \in I} \lambda_{i} m_{i} \text {, for some }\left\{\lambda_{i}\right\}_{i \in I}\right\} .
$$

Said in other words, $M$ is the direct sum of subspaces $\left\{M_{i}\right\}_{i \in I}$ :

$$
M=\bigoplus_{i \in I} M_{i}
$$

where $M_{i}=\left\langle m_{i}\right\rangle$ is the subspace of $M$ generated by $m_{i}$. In algebraic terms, $M$ is the subset of $\prod_{i \in I} M_{i}$ where only finitely many terms are nonzero. Of course, the condition that $M$ is a subspace supposes a frictionless trading, that is to say, unlimited short sales without transaction costs. 
4. The market assigns a price $p_{i}$ to the marketed claim $m_{i}$, for every $i \in I$. It is not necessary to suppose that prices are a priori positive, but rather this property can be deduced as a consequence of the absence of arbitrage opportunities [4]. A price correspondence $p: M \rightarrow \mathbb{R}$ is naturally defined by the formula:

$$
p(m)=\left\{\sum_{i \in I} \lambda_{i} p_{i}: m=\sum_{i \in I} \lambda_{i} m_{i}, \text { for some }\left\{\lambda_{i}\right\}_{i \in I}\right\},
$$

for every $m \in M$. The economic interpretation of this price can be found in [4].

Observe that this model is wide enough to consider the possibility that $p$ is not even a map but simply a correspondence. Let us see several examples of real situations. For other situations of arbitrage, in the context of financial engineering, see [13].

Example 1. Figure 2 describes an arbitrage and gives a graphical visualization of the so-called triangular arbitrage (a bid price is the highest price that a buyer is willing to pay for a good; on the other hand, an ask price is the price that a seller states s/he will accept; ask is always greater than bid). In effect, observe that:

$$
S_{€ / \$}^{b i d} \times S_{€ / £}^{a s k} \neq S_{\$ / £^{\prime}}^{b i d}
$$

which indicates the presence of an arbitrage profit. For a detailed calculation, see Example 2.

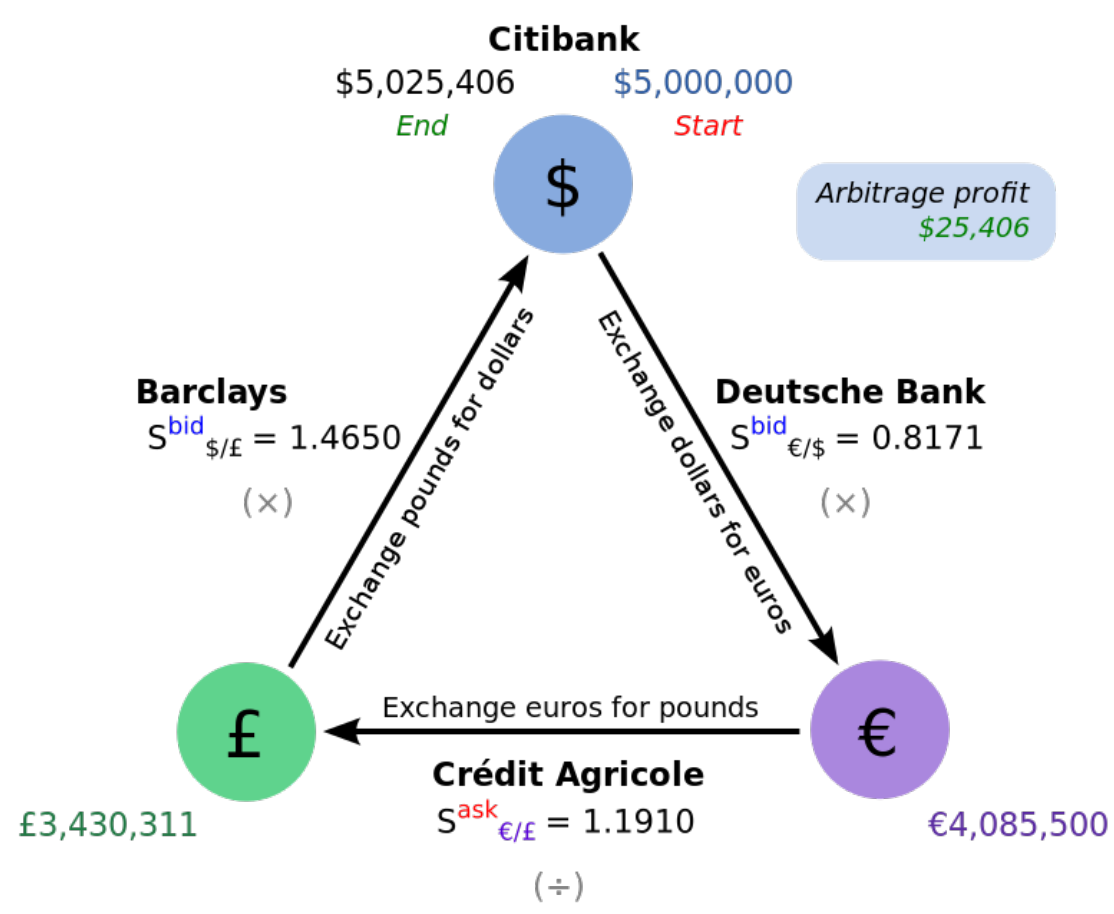

Figure 2. Triangular arbitrage. Source: [14].

Example 2. Another triangular arbitrage is presented in Table 2.

Table 2. Cross exchange rates. Source: [14].

\begin{tabular}{cccc}
\hline Coin & USD & EUR & GBP \\
\hline USD & 1.00000 & 0.89347 & 0.76988 \\
EUR & 1.11923 & 1.00000 & 0.86167 \\
GBP & 1.29891 & 1.16054 & 1.00000 \\
\hline
\end{tabular}


Let us consider the following contingent claims:

- $\quad$ change US\$1.00 into $€ 0.89347$,

- $\quad$ change $€ 0.89347$ into $£ 0.89347 \times 0.86167=£ 0.769876295$, and

- $\quad$ change back $£ 0.769876295$ into US\$0.769876295 × $1.2981=U S \$ 0.999376418$,

which would actually result into a small loss. Alternatively, one could:

- $\quad$ change US\$1.00 into £0.76988,

- $\quad$ change $£ 0.76988$ into $€ 1.16054 \times 0.76988=€ 0.893476535$, and

- $\quad$ change back $€ 0.893476535$ into US\$0.893476535 × $1.11923=U S \$ 1.000005742$,

which would result into a small gain, assuming the absence of transaction costs. In this example, one has the following contingent claims:

- $m_{1}$ : "change US\$1.00 into $€$ ",

- $m_{2}$ : "change $€$ into $£^{\prime \prime}$, and

- $m_{3}:$ "change US\$1.00 into $£^{\prime \prime}$,

with respective prices:

- $\quad p_{1}=0.89347$,

- $\quad p_{2}=0.86167$, and

- $\quad p_{3}=0.76988$.

Taking $\lambda_{3}=5,000,000.00$, the number $\lambda_{2}$ of equivalent contingent claims $m_{2}$ is given by solving the following equation:

$$
\begin{aligned}
5,000,000.00 m_{3} & =\lambda_{2} m_{2}, \\
5,000,000.00 \times 0.76988 & =\lambda_{2} \times 0.86167,
\end{aligned}
$$

from where $\lambda_{2}=4,467,371.50$. However, the prices do not coincide:

$$
\lambda_{3} p_{3}=5,000,000 \times 1=5,000,000.00
$$

and

$$
\lambda_{2} p_{2}=4,467,371.50 \times 1.11923=5,000,016.20 .
$$

In the next sections, we will justify that this difference is due, among other reasons, to the absence of transitivity as a necessary (not sufficient) condition to reach a unique price.

Thus, it is just possible that the same marketed claim has different prices according to its specific linear expression. This is because $p: M \rightarrow \mathbb{R}$ is a linear correspondence. However, this is equivalent to requiring that

$$
p: M \rightarrow 2^{\mathbb{R}}
$$

is a linear map (also, we can use $\mathcal{P}(\mathbb{R})$ or, more specifically, $\mathbb{R}\{\mathbb{R}\}$ ) with the operations:

$$
A+B:=\{a+b: a \in A, b \in B\}
$$

and

$$
\lambda A:=\{\lambda a: a \in A\},
$$

where $A \in 2^{\mathbb{R}}, B \in 2^{\mathbb{R}}$, and $\lambda \in \mathbb{R}$. Observe that the initial correspondence and the subsequent map have been both denoted by $p$.

This justifies the following binary relation on $M$ : For every $m$ and $m^{*}$ in $M, m \sim_{p} m^{*}$ if, and only if, $p(m)=p\left(m^{*}\right)$. As $p(m)$ and $p\left(m^{*}\right)$ are allowed to be subsets of $\mathbb{R}, \sim_{p}$ is an equivalence relation on $M$. This is the well-known equivalence relation associated to a map. 
Suppose that, in general, $M$ is a linear subspace of $X$. A linear map $p: M \rightarrow \mathbb{R}$ is said to be positive provided that $p(x) \geq 0$, for every $x \in C \cap M$; and it is strictly positive provided that $p(x)>0$, for every $x \in C \cap M$.

We will always assume that $C \cap M \neq \varnothing$ and that there exists a distinguished vector $m_{0}$ in $C \cap M$ such that $p\left(m_{0}\right)>0$. (In general, given a vector $m, p(m)>0$ means $c>0$, for every $c \in p(m)$. However, Corollary 1 will show that $p\left(m_{0}\right)$ is a unique positive real number (or a unitary set composed by a positive real number).) The strictly positive contingent claim $m_{0}$ is interpreted as a government bond (fiat money, i.e., a short-term riskless bond paying no dividends, as a numéraire). In particular, $m_{0}$ could be the claim which pays 1 unit of the numéraire in each state of the world, denoted by 1 .

\section{NA Condition Involving the Preference Relation}

There are lot of descriptions of arbitrage situations. In this section, we highlight the definition provided by [15], "an arbitrage opportunity never loses any wealth and with strictly positive probability it generates positive wealth at time $T^{\prime \prime}$. Following with the description of our setting, let us denote by $F:=\{m \in M: c \leq 0$, for some $c \in p(m)\}$ the set of feasible contingent claims. Based on his seminal papers [16,17], the following definition of NA was originally proposed by Ross [18]: A simple free lunch is a feasible claim which is strictly positive. The principle of NA states that there is no simple free lunch, i.e.:

$$
\text { NA: } C \cap F=\varnothing \text {. }
$$

This condition is equivalent to require that $C \cap F_{0}=\varnothing$, where $F_{0}:=\{m \in M: p(m)=0\}=\operatorname{ker} p$. (In effect, if $p(m) \leq 0$, there exists $\alpha \geq 0$ such that $p\left(m+\alpha m_{0}\right)=0$. By hypothesis, $m+\alpha m_{0}=0$ and so $m=-\alpha m_{0} \preceq 0$. So, $m \notin C$.) (In general, given a vector $m, p(m)=0$ means that $p(m)$ is the unitary set composed by the real number 0 .) On the other hand, it is well known that NA holds if and only if the linear map price $p$ is strictly positive [4]:

$$
\text { For every } m \in M, m \succ 0 \text { implies } p(m)>0 \text {. }
$$

Observe that the former condition is equivalent to the following statement:

For every $m_{1}$ and $m_{2} \in M, m_{1} \succ m_{2}$ implies $p\left(m_{1}\right)>p\left(m_{2}\right)$.

Now, starting from the quotient space $M / \sim_{p}$, let us consider the following map:

$$
\bar{p}:\left(M / \sim_{p}\right) \rightarrow 2^{\mathbb{R}},
$$

given by:

$$
\bar{m} \mapsto \bar{p}(\bar{m}):=p(m),
$$

which is obviously well defined. In order to know the cardinality of $\bar{p}(\bar{m})$ or $p(m)$, let us start from the following lemma.

Lemma 1. For every $m \in M, \operatorname{card}(p(m))=1 \operatorname{or} \operatorname{card}(p(m))=\aleph_{0}$.

Proof. Given an $m \in M$, let us assume that $\operatorname{card}(p(m)) \neq 1$. In this case, we can choose two different elements $c$ and $c^{*}$ belonging to $p(m)$. Moreover, $m$ can be written by using two different linear combinations $\left\{m_{i}\right\}_{i \in I}$ with coefficients $\left\{\lambda_{i}\right\}_{i \in I}$ and $\left\{\lambda_{i}^{*}\right\}_{i \in I}$ :

$$
m=\sum_{i \in I} \lambda_{i} m_{i}=\sum_{i \in I} \lambda_{i}^{*} m_{i}
$$

such that

$$
c=\sum_{i \in I} \lambda_{i} p_{i},
$$

and

$$
c^{*}=\sum_{i \in I} \lambda_{i}^{*} p_{i}
$$


For every $\alpha \in \mathbb{R}$, one has:

$$
m=\alpha m+(1-\alpha) m=\sum_{i \in I}\left[\alpha \lambda_{i}+(1-\alpha) \lambda_{i}^{*}\right] m_{i} .
$$

So,

$$
\begin{gathered}
p(m) \ni \sum_{i \in I}\left[\alpha \lambda_{i}+(1-\alpha) \lambda_{i}^{*}\right] p_{i}= \\
=\alpha\left(\sum_{i \in I} \lambda_{i} p_{i}\right)+(1-\alpha)\left(\sum_{i \in I} \lambda_{i}^{*} p_{i}\right)=\alpha c+(1-\alpha) c^{*} .
\end{gathered}
$$

Observe that, for every $x \in \mathbb{R}$, there is an $\alpha$ such that $x=\alpha c+(1-\alpha) c^{*}$. In effect, it suffices to take

$$
\alpha=\frac{x-c^{*}}{c-c^{*}} .
$$

Therefore, $p(m)=\mathbb{R}$.

Corollary 1. The set of feasible contingent claims, F, includes all vectors whose image is either $\mathbb{R}$ or a unique non-positive price. On the other hand, the image of all vectors in $C$ with a positive price (e.g., $m_{0}$ ) is unique.

Next, we are going to introduce a little of algebraic structure. Let $U$ be the subset of $M$ defined as:

$$
U=\{u \in M: \operatorname{card}(p(u))=1\} .
$$

The sum is closed in $U$. In effect, assume that $u$ and $u^{*} \in U$. Then

$$
\begin{gathered}
u=\sum_{i \in I} \lambda_{i} m_{i}, \\
u^{*}=\sum_{i \in I} \lambda_{i}^{*} m_{i}, \\
p(u)=\sum_{i \in I} \lambda_{i} p_{i},
\end{gathered}
$$

and

$$
p\left(u^{*}\right)=\sum_{i \in I} \lambda_{i}^{*} p_{i}
$$

By the definition of $p$,

$$
p\left(u+u^{*}\right) \ni \sum_{i \in I}\left(\lambda_{i}+\lambda_{i}^{*}\right) p_{i} .
$$

Assume that, moreover,

$$
p\left(u+u^{*}\right) \ni \sum_{i \in I} \mu_{i} p_{i} .
$$

Thus,

$$
p(u) \ni \sum_{i \in I}\left(\mu_{i}-\lambda_{i}^{*}\right) p_{i}
$$

from where, as $u \in U, \mu_{i}-\lambda_{i}^{*}=\lambda_{i}$ and then $\mu_{i}=\lambda_{i}+\lambda_{i}^{*}$. An analogous reasoning could be made for the product by a scalar. In particular, we can deduce that $0 \in U$ and, consequently, $U$ is a vector subspace of $M$.

Finally, the subset $V$ of $M$ defined as:

$$
V=\left\{v \in M: \operatorname{card}(p(v))=\aleph_{0}\right\}
$$


is a vector semispace of $M$. Obviously, $U \cup V=M$ and $U \cap V=\varnothing$.

At this point, we are going to introduce a scheme (see Figure 3) whose aim is to provide a general view of the sets involved in our setting.

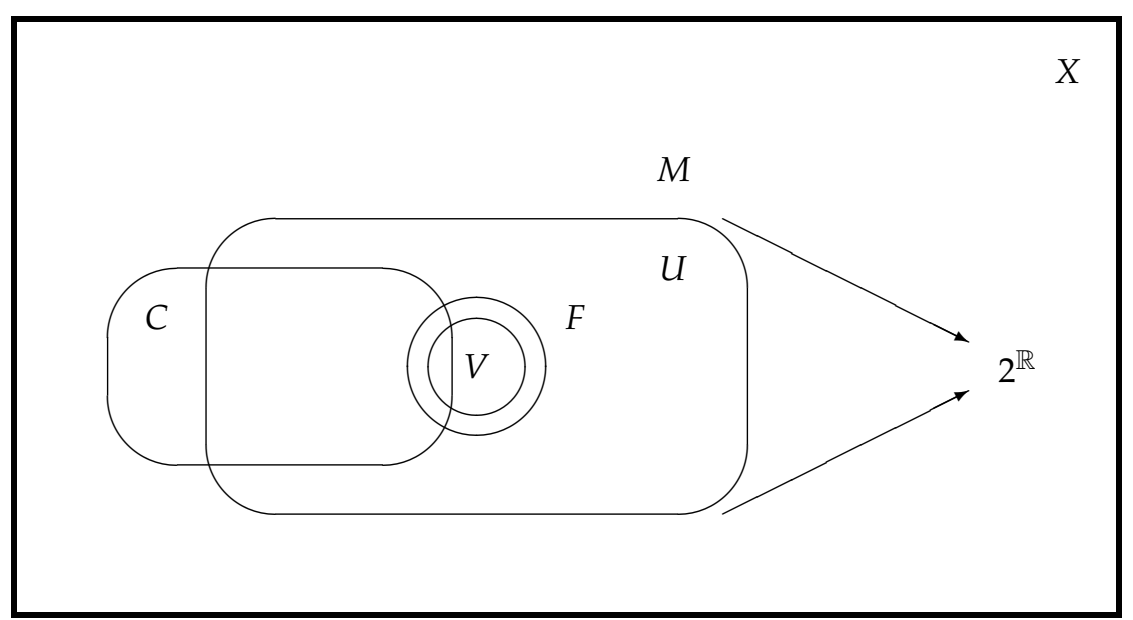

Figure 3. Diagram with the main sets in the arbitrage theory. Source: Own elaboration.

Figure 4 clarifies the cardinality of $p(m)$ according to its origin. Our aim is to arrive to the situation described by Figure 5 .

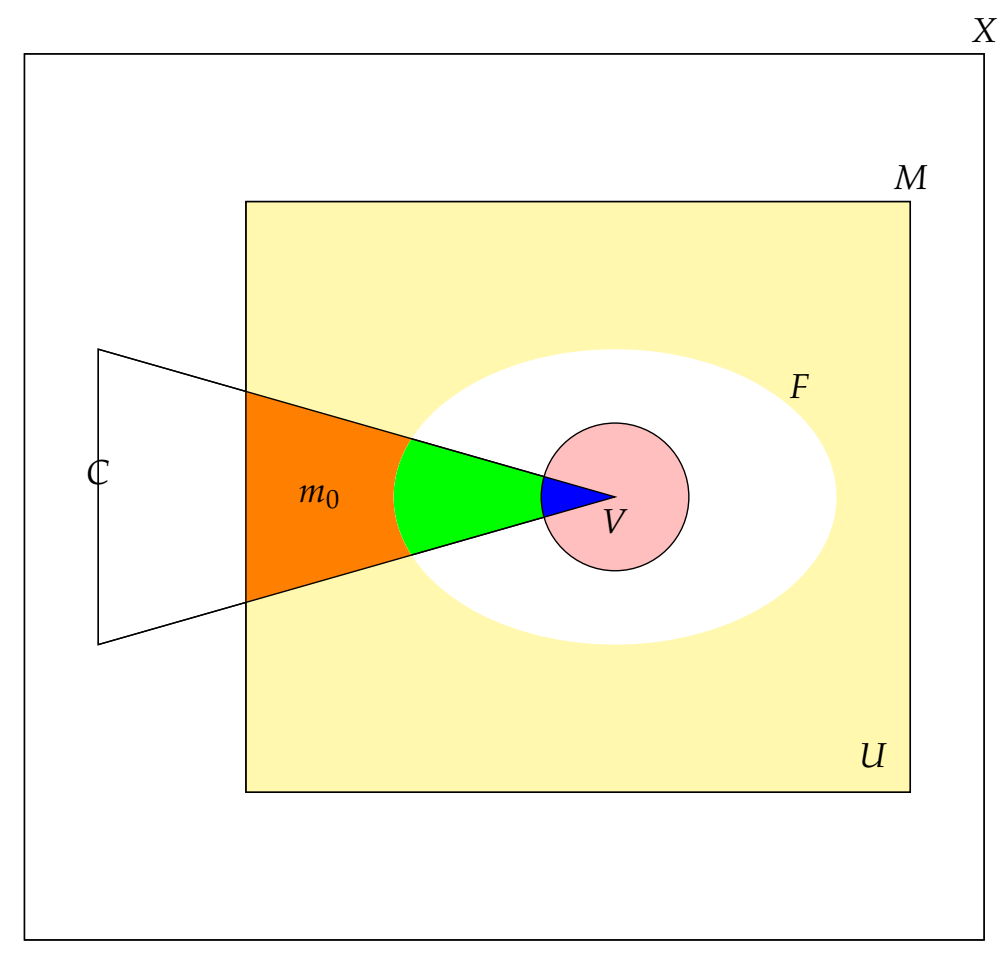

Figure 4. In yellow, elements in $M$ with a unique (positive) price not in $C$. In orange, elements in $C \cap M$ with a unique (positive) price. In green, elements in $C \cap F$ with a unique (negative or null) price. In blue, elements in $C \cap F$ with infinite prices. In pink, elements in $F$ with infinite prices not in $C$. In white, elements in $F$ with a unique (negative or null) price. Source: Own elaboration. 


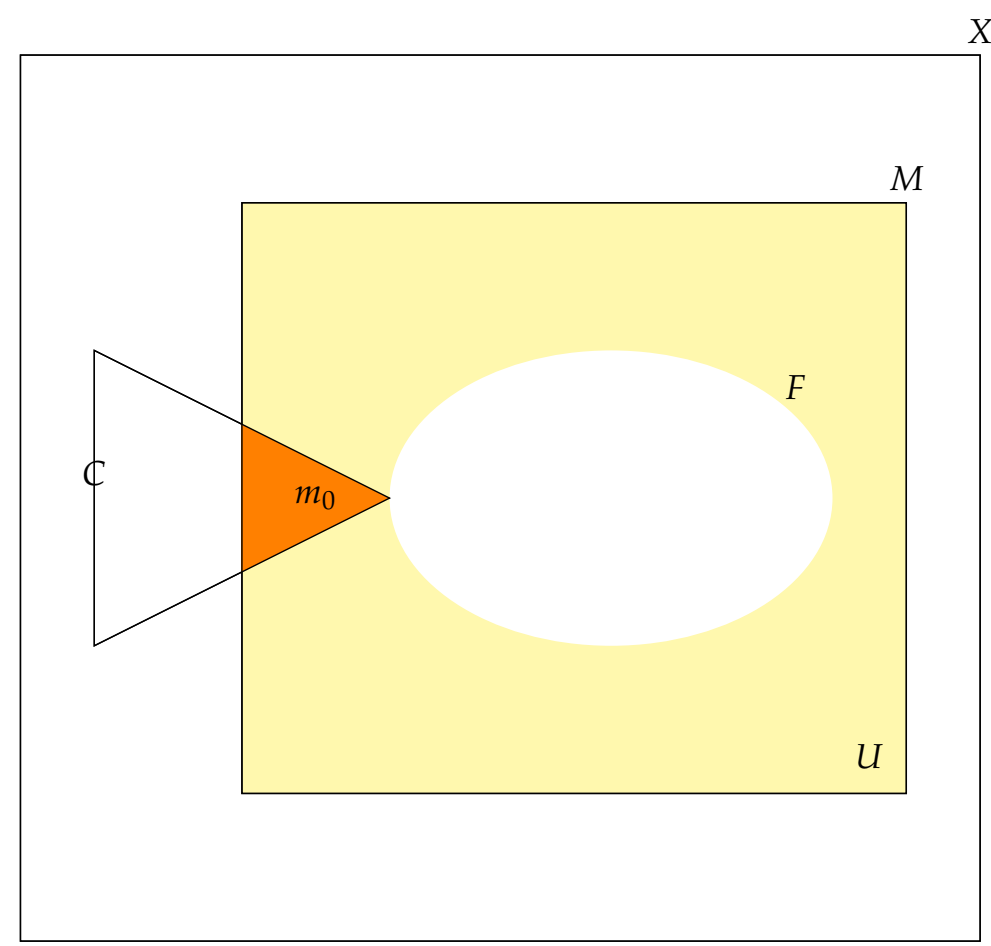

Figure 5. In yellow, elements in $M$ with a unique (positive) price. In orange, elements in $C \cap M$ with a unique (positive) price. In white, elements in $F$ with a unique (negative or null) price. Source: Own elaboration.

Theorem 1 provides a characterization of the NA condition by only requiring some restrictions on the involved binary relation, but first we are going to introduce the following definitions. In spite of the fact that our model allows unlimited short and long positions, Definition 1 reflects the idea that, in real decision-making, investors set upper and lower bounds, respectively, to theoretical positions.

Definition 1 ([19]). An ordering relation $\succeq$ is Archimedean if, for every $x_{1}$ and $x_{2} \in X$, there exist $\alpha$ and $\beta$ such that

$$
\alpha x_{1} \succeq x_{2} \succeq \beta x_{1} .
$$

Definition 2 ([15]). An ordering relation $\succeq$ satisfies the axiom of the "intermediate value" if, for every $x_{1}, x_{2}$ and $x_{3} \in X$, such that $x_{1} \succeq x_{3} \succeq x_{2}$, there exists $\gamma \in[0,1]$ such that

$$
x_{3} \sim\left[\gamma x_{1}+(1-\gamma) x_{2}\right] .
$$

Definition 3 ([15]). An ordering relation $\succeq$ is complete or satisfies the axiom of the "comparability" if, for every $x_{1}$ and $x_{2} \in X$, either $x_{1} \succeq x_{2}$ or $x_{2} \succeq x_{1}$.

In Section 3, we will introduce two definitions analogous to Definitions 1 and 3.

On $\left(M / \sim_{p}\right)$ we define the following binary relation: $\bar{m} \succeq^{*} \bar{n}$ if, for every $z \in \bar{m}$ and $w \in \bar{n}, z \nprec w$ holds. This can also be said as " $\bar{m}$ dominates $\bar{n} "$.

Theorem 1. The following four conditions are equivalent:

(i) NA condition.

(ii) $p$ is a map and $\succeq^{*}$ is complete on $M / \sim_{p}$.

(iii) $p$ is a map and $\left(M / \sim_{p}\right) \cong\left\langle\overline{m_{0}}\right\rangle$.

(iv) $\succeq *$ is Archimedean and satisfies the axiom of the intermediate value. 
Proof. (i) $\Rightarrow$ (ii). For every $m \in M$, assume that $c$ and $c^{*}$, such that $c<c^{*}$, belongs to $p(m)$. Then, there exists $\left\{\lambda_{i}\right\}_{i \in I}$ and $\left\{\lambda_{i}^{*}\right\}_{i \in I}$ such that:

$$
\begin{gathered}
m=\sum_{i \in I} \lambda_{i} m_{i}=\sum_{i \in I} \lambda_{i}^{*} m_{i}, \\
c=\sum_{i \in I} \lambda_{i} p_{i}
\end{gathered}
$$

and

$$
c^{*}=\sum_{i \in I} \lambda_{i}^{*} p_{i}
$$

One has:

$$
0=m-m=\sum_{i \in I}\left(\lambda_{i}-\lambda_{i}^{*}\right) m_{i}
$$

from where

$$
p(0) \ni c-c^{*}<0 .
$$

(Taking into account that $0 \in U$, we can also write $p(0)=c-c^{*}<0$.)

Let $m_{0} \in M^{++}$. By hypothesis, $p\left(m_{0}\right)>0$. Let us consider the vector $z:=\frac{c^{*}-c}{p_{0}} m_{0}$, where $p_{0}=p\left(m_{0}\right)$. Obviously, $z \in C$ and, moreover:

$$
p(z)=p(z+0)=\frac{c^{*}-c}{p_{0}} p_{0}+c-c^{*}=0 .
$$

Therefore, $z \in F$ and then $C \cap F \supseteq\{z\}$ and so the NA condition does not hold. Thus, $c=c^{*}$ and $p$ is a map.

On the other hand, let us consider two equivalence classes $\bar{m}$ and $\bar{n}$. There is no loss of generality in assuming that $p(\bar{m}) \geq p(\bar{n})$ and take $\alpha$ and $\beta, \alpha \geq \beta$, such that:

$$
\alpha p\left(m_{0}\right)=p\left(\alpha m_{0}\right):=p(\bar{m}),
$$

and

$$
\beta p\left(m_{0}\right)=p\left(\beta m_{0}\right):=p(\bar{n}) .
$$

Then $\overline{\alpha m_{0}}=\bar{m}$ and $\overline{\beta m_{0}}=\bar{n}$. Taking into account that $\alpha m_{0} \succeq \beta m_{0}$ and that it is not possible the existence of a $z \in \bar{m}$ and an $w \in \bar{n}$ such that $z \prec w$ (which would imply $p(z)<p(w)$ ), it can be deduced that $\bar{m} \succeq * \bar{n}$.

(ii) $\Rightarrow$ (iii). For every $\bar{m} \in\left(M / \sim_{p}\right)$, by hypothesis, $\bar{m} \succeq^{*} \overline{m_{0}}$ or $\overline{m_{0}} \succeq^{*} \bar{m}$. If $\bar{m} \succeq^{*} \overline{m_{0}}$, then, as $p$ is a map, $p(\bar{m}) \geq p\left(\overline{m_{0}}\right)$. Take $\alpha \geq 1$ such that $p(\bar{m})=\alpha p\left(\overline{m_{0}}\right)$. Therefore, $\bar{m}=\overline{\alpha m_{0}}=\alpha \overline{m_{0}}$.

Otherwise, if $\overline{m_{0}} \succeq^{*} \bar{m}$, the reasoning is analogous with $\alpha \leq 1$.

(iii) $\Rightarrow$ (iv). For every $\bar{m}$ and $\bar{n} \in\left(M / \sim_{p}\right)$, by hypothesis, one has:

$$
\bar{m}=\overline{\alpha m_{0}}, \alpha \in \mathbb{R},
$$

and

$$
\bar{n}=\overline{\beta m_{0}}, \beta \in \mathbb{R} .
$$

There is no loss of generality in assuming that $\alpha$ and $\beta$ have the same sign. In this case, there exists $k \in \mathbb{R}, k \neq 0$, such that

$$
k \alpha \geq \beta \geq \frac{\alpha}{k} .
$$

Consequently,

$$
k \bar{m}=\overline{k \alpha m_{0}} \succeq * \overline{\beta m_{0}}=\bar{n} \succeq * \bar{\alpha} \frac{\bar{\alpha} m_{0}}{k}=\frac{1}{k} \bar{m}
$$


and so

$$
k \bar{m} \succeq * \bar{n} \succeq^{*} \frac{1}{k} \bar{m}
$$

whereby $\succeq^{*}$ is Archimedean on $M / \sim_{p}$.

On the other hand, let us assume that $\overline{m_{1}} \succeq^{*} \bar{n} \succeq^{*} \overline{m_{2}}$. By hypothesis, one has:

$$
\begin{aligned}
& \overline{m_{1}}=\overline{\alpha_{1} m_{0}}, \alpha_{1} \in \mathbb{R}, \\
& \overline{m_{2}}=\overline{\alpha_{2} m_{0}}, \alpha_{2} \in \mathbb{R},
\end{aligned}
$$

and

$$
\bar{n}=\overline{\beta m_{0}}, \beta \in \mathbb{R} .
$$

In this case, there exists $\lambda \in \mathbb{R}, 0 \leq \lambda \leq 1$, such that

$$
\lambda \alpha_{1}+(1-\lambda) \alpha_{2}=\beta .
$$

Consequently,

$$
\lambda \overline{m_{1}}+(1-\lambda) \overline{m_{2}}=\bar{n}
$$

and so $\succeq^{*}$ satisfies the axiom of the intermediate value on $\left(M / \sim_{p}\right)$.

(iv) $\Rightarrow$ (i). Assume that $m \succ 0$. As $\succeq^{*}$ is Archimedean on $M / \sim_{p}$, there exist $\alpha$ and $\beta \in \mathbb{R}$ such that:

$$
\alpha \overline{m_{0}} \succeq^{*} \bar{m} \succeq^{*} \beta \overline{m_{0}} .
$$

Then, by the axiom of the intermediate value on $M / \sim_{p}$, there exists $\gamma, \alpha \geq \gamma \geq \beta$, such that:

$$
\bar{m}=\overline{\gamma m_{0}} \text {. }
$$

Consequently, $p(\bar{m})>0$ and, in particular, $p(m)>0$.

Finally, a geometric interpretation of the absence of arbitrage is provided by Figure 6 . If the NA condition holds, it is well known that $p: M \rightarrow \mathbb{R}$ is a strictly increasing linear map, so

$$
\bar{p}:(M / \operatorname{ker} p) \rightarrow \mathbb{R}
$$

is an increasing monomorphism of the quotient space composed by the indifference hyperplanes:

$$
H_{k}=\left\{x: x=\sum_{i \in I} \lambda_{i} m_{i}, \sum_{i \in I} \lambda_{i} p_{i}=k, \text { for some }\left\{\lambda_{i}\right\}_{i \in I}\right\} .
$$


$m_{2}$

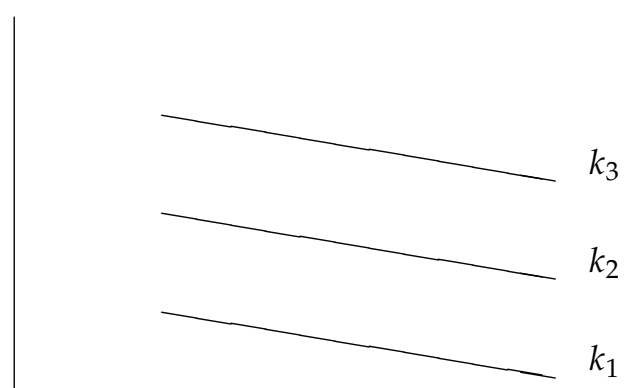

$m_{1}$

Figure 6. Indifference lines (case in which $I=\{1,2\}$ ). Source: Own elaboration.

\section{NA Condition Involving the Cone}

As indicated in the Introduction, the cone $C$ is not necessarily convex. However, the following proposition provides four equivalent conditions which are necessary when requiring the NA condition.

Proposition 1. The following four conditions are equivalent:

(i) $C \cup\{0\}$ is a convex cone.

(ii) For every $x \in C \cup\{0\}$, the affine cone $C_{x}:=\left\{x^{\prime} \in C: x^{\prime} \succeq x\right\}$ is closed for the sum.

(iii) $\succeq$ is convex.

(iv) $\succeq$ is transitive.

Proof. The equivalence (i) $\Leftrightarrow$ (iv) was discussed in the Introduction.

(i) $\Leftrightarrow$ (ii). In effect, consider two arbitrary elements $x^{\prime}, x^{\prime \prime} \succeq x$. As $x-\frac{1}{2} x=\frac{1}{2} x \in C \cup\{0\}$, then $x \succeq \frac{1}{2} x$. So, by the transitivity of $\succeq$, one has:

$$
x^{\prime} \succeq \frac{1}{2} x
$$

Analogously, it can be shown that:

$$
x^{\prime \prime} \succeq \frac{1}{2} x
$$

By the definition of $\succeq$, it is verified that:

$$
x^{\prime}-\frac{1}{2} x \in C \cup\{0\} \text { and } x^{\prime \prime}-\frac{1}{2} x \in C \cup\{0\}
$$

and, since $C \cup\{0\}$ is convex,

$$
x^{\prime}+x^{\prime \prime}-x \in C \cup\{0\}
$$

and so $x^{\prime}+x^{\prime \prime} \succeq x$.

The converse implication is obvious because $C \cup\{0\}=C_{0}$.

(i) $\Leftrightarrow$ (iii). In effect, assume that $C \cup\{0\}$ is convex. Let us consider three arbitrary elements $x$, $x^{\prime}$ and $x^{\prime \prime}$, with $x, x^{\prime} \succeq x^{\prime \prime}$. 
As $x \succeq x^{\prime \prime}$, by the definition of $\succeq, x-x^{\prime \prime} \in C \cup\{0\}$. For every $\lambda \in[0,1]$, by the definition of $C \cup\{0\}$, $\lambda\left(x-x^{\prime \prime}\right) \in C \cup\{0\}$. Analogously, from $x^{\prime} \succeq x^{\prime \prime}$, we can deduce that $(1-\lambda)\left(x^{\prime}-x^{\prime \prime}\right) \in C \cup\{0\}$. Thus, by hypothesis $(C \cup\{0\}$ is convex), one has:

$$
\lambda\left(x-x^{\prime \prime}\right)+(1-\lambda)\left(x^{\prime}-x^{\prime \prime}\right) \in C \cup\{0\} .
$$

Making some operations and rearranging terms:

$$
\lambda x+(1-\lambda) x^{\prime}-x^{\prime \prime} \in C \cup\{0\},
$$

from where:

$$
\lambda x+(1-\lambda) x^{\prime} \succeq x^{\prime \prime}
$$

and so $\succeq$ is convex.

Reciprocally, assume that $\succeq$ is convex. Let us consider two arbitrary elements $x$ and $x^{\prime}$ in $C \cup\{0\}$. By the definition of $\succeq$, one has $x, x^{\prime} \succeq 0$ and, by hypothesis ( $\succeq$ is convex):

$$
\frac{1}{2} x+\frac{1}{2} x^{\prime} \succeq 0
$$

By the definition of $\succeq, \frac{1}{2} x+\frac{1}{2} x^{\prime} \in C \cup\{0\}$ and, by the definition of $C \cup\{0\}$,

$$
2\left(\frac{1}{2} x+\frac{1}{2} x^{\prime}\right) \in C
$$

or, what is the same,

$$
x+x^{\prime} \in C \cup\{0\}
$$

and so $C \cup\{0\}$ is convex.

Next, we are going to introduce a characterization for the NA condition.

Theorem 2. NA holds if, and only if, $(C \cap M) / \operatorname{ker} p=-(F / \operatorname{ker} p)^{*}$. (From now on, given a set $A, A^{*}$ will be the set $A$ without the null element.)

Proof. First, assume that NA holds. For every $m \in F \backslash(\operatorname{ker} p), p(m)<0$ and then there exists at least an element $m^{*} \in M$ such that

$$
p\left(m^{*}\right)=-p(m) .
$$

In effect, take the real number $\lambda$ such that:

$$
p\left(\lambda m_{0}\right)=-p(m)>0 .
$$

As $p$ is a linear map,

$$
\lambda p\left(m_{0}\right)=-p(m)
$$

and so

$$
\lambda=-\frac{p(m)}{p\left(m_{0}\right)}>0 .
$$

Note that this quotient makes sense since $p\left(m_{0}\right)$ is strictly positive. Now, $m_{0} \in C \cap M$ which implies that $m^{*}:=\lambda m_{0} \in C \cap M\left(\lambda m_{0} \in C\right.$ since $C$ is a cone and $\lambda m_{0} \in M$ because $M$ is a vector subspace). Therefore, $p\left(m+m^{*}\right)=0$ and so $m+m^{*} \in \operatorname{ker} p$. Consequently, $\overline{m^{*}}=-\bar{m}$.

The proof of the other inclusion is analogous. 
Reciprocally, assume that $(C \cap M) / \operatorname{ker} p=-(F / \operatorname{ker} p)^{*}$. In this case, $C \cap F=\varnothing$, because if $C \cap F \neq \varnothing$, then there exists $c \succ 0, c \in M$, such that $p(c) \leq 0$. If $p(c)=0$, then $c \in$ ker $p$ and so $\overline{0} \in(C \cap M) / \operatorname{ker} p$ but this is not possible because $\overline{0} \notin-(F / \operatorname{ker} p)^{*}$. Therefore, $p(c)<0$.

By hypothesis, we can find $c^{*} \in(F \backslash \operatorname{ker} p)$, such that $c+c^{*} \in \operatorname{ker} p$ from which $p\left(c+c^{*}\right)=0$ and then $p(c)=-p\left(c^{*}\right) \geq 0$. This supposes a contradiction which comes from the assumption $C \cap F \neq \varnothing$; hence $C \cap F=\varnothing$ and NA holds.

As shown, if NA holds, $m \succ 0$ implies $p(m)>0$. However, the converse implication does not necessarily hold. This is because, after requiring the NA condition, the elements of a part of $M$ can have strictly positive prices (in Figure 4, in yellow). So, we wonder what should be the structure of the cone in order to reach the following statement:

For every $m \in M, m \succ 0$ if, and only if, $p(m)>0$.

Obviously, in general $C \supseteq\left\{\lambda m_{0}\right\}_{\lambda>0}$. It is straightforward to show that this more restricted NA condition holds if, and only if, $C=\left\{\lambda m_{0}\right\}_{\lambda>0}+\operatorname{ker} p$, which is the maximum cone. On the other hand, the minimum cone $C$ is the ray $\left\{\lambda m_{0}\right\}_{\lambda>0}$.

In order that a new cone (Figure 7) makes sense, it must be included in the maximum cone. In effect, a new cone $K$ satisfies $K \cap F=\varnothing$ if, and only if, $K \subseteq M \backslash F=C+\operatorname{ker} p$. The role of $K$ is to extend the preference relation $\succeq$ making it coincide with $\succeq_{p}$. In effect, the following construction generalizes arbitrage situations. Let $D$ be a subset of $M$, such that $0 \notin D$. In a beginning, the price of an element of $D$ can be positive, negative or zero. The set of all positive scalar multiples of $D$ :

$$
K:=\bigcup_{\lambda>0} \lambda D
$$

is, obviously, a cone. ([5] call desirable the elements of $D$, virtually desirable the elements of $K$, good deal the elements of $D \cap F$, and virtually good deal the elements of $K \cap F$.) The pointed cone $K \cup\{0\}$ gives rises to an ordering in $M$ by putting $x \succeq y$ if $x-y \in K \cup\{0\}$ and $x \succ y$ if $x-y \in K$. Now, we wonder what are the consequences of considering this cone instead of $C$. Theorem 6 [5] shows that $K \cap F=\varnothing$ (that is to say, the absence of virtually good deals) implies that $p: M \rightarrow \mathbb{R}$ is a $K$-strictly positive linear functional, i.e., $p(m)$ is unique for every $m \in M, p\left(m_{1}+m_{2}\right)=p\left(m_{1}\right)+p\left(m_{2}\right)$, for every $m_{1} \in M$ and $m_{2} \in M$, and $p$ assigns strictly positive prices to elements of $K$.

Summarizing, two situations can occur:

1. $K \subseteq\left\{\lambda m_{0}\right\}_{\lambda>0}+(\operatorname{ker} p)^{*}$, which implies $K=\left\{\lambda m_{0}\right\}_{\lambda>0}+J^{*}$, where $J^{*}$ is a cone included in $(\operatorname{ker} p)^{*}$. In this case, $K \cap\left\{\lambda m_{0}\right\}_{\lambda>0}=\varnothing$.

2. $K \subseteq\left\{\lambda m_{0}\right\}_{\lambda>0}+\operatorname{ker} p$, which implies $K=\left\{\lambda m_{0}\right\}_{\lambda>0}+J$, where $J$ is a cone included in $\operatorname{ker} p$. In this case, $K \supseteq\left\{\lambda m_{0}\right\}_{\lambda>0}$. 


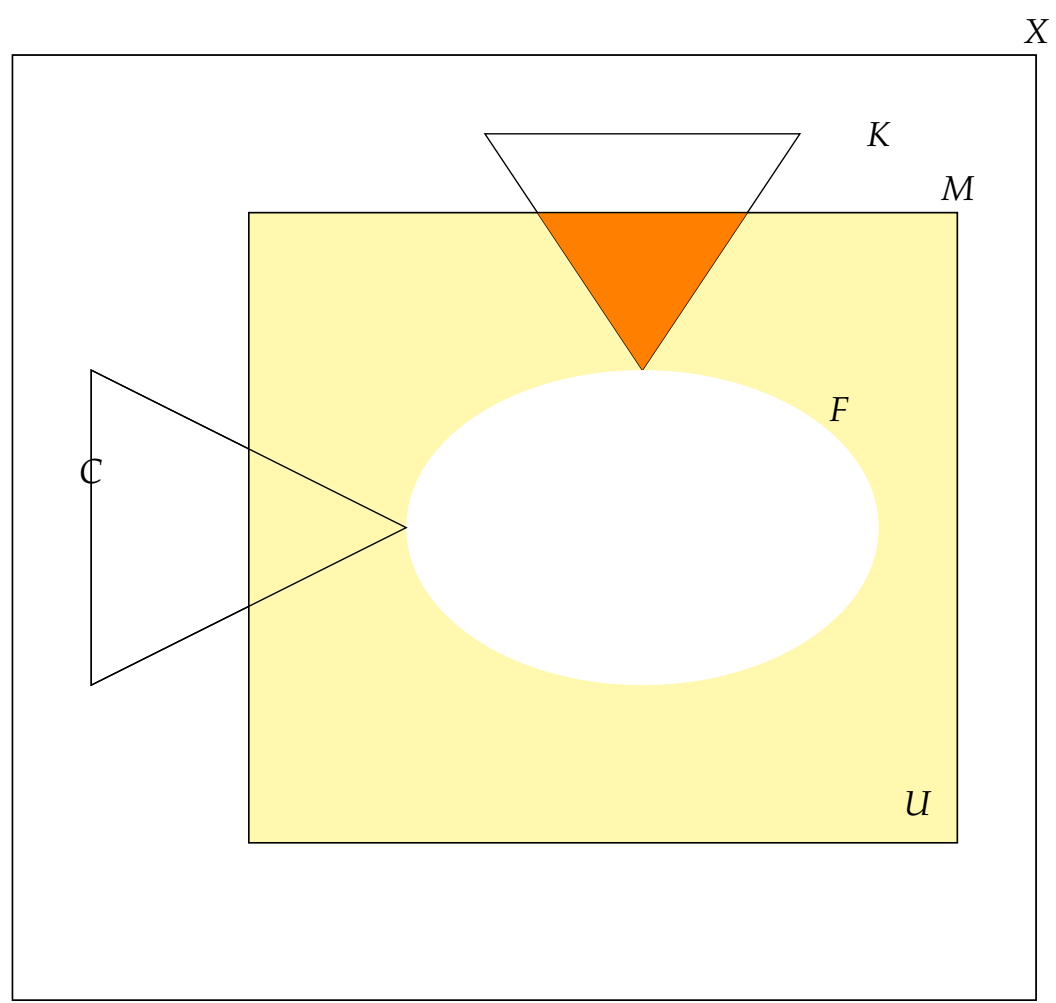

Figure 7. In yellow, elements in $M$ with a unique (positive) price. In orange, elements in $K \cap M$ with a unique (positive) price. In white, elements in $F$ with a unique (negative or null) price. Source: Own elaboration.

Inside this second case, a noteworthy family of sets $D$ is given by the affine cones:

$$
D(a):=\left\{x \in X: x \succeq a m_{0}\right\}=a m_{0}+C,
$$

where $a \geq 0$ is called the desirability level. The cone generated by $D(a)$ is (Figure 8):

$$
K(a):=\bigcup_{\lambda>0} \lambda D(a)
$$

If $C$ is convex, one has:

$$
C=K(0) \supseteq K(a) \supseteq K(b),
$$

provided that $0<a<b$. In this case, one has:

$$
C \supseteq \bigcap_{a \geq 0} K(a) .
$$

In general, the converse statement is not true, whereby, in order to obtain some equivalences, we are going to introduce some necessary definitions [5]. First, we are going to introduce a necessary and sufficient condition for the reverse inclusion.

Definition 4. The preference relation $\succeq$ is said to be arbitrage-sensitive if, for every desirability level $a \geq 0$ and every $x \in C$, there exists $\mu$ large enough such that $\mu x \succeq$ amo.

We can enunciate the following statement.

Proposition 2. The preference relation $\succeq$ is arbitrage-sensitive if, and only if, $C \subseteq \bigcap_{a \geq 0} K(a)$. 
Proof. First, assume that the preference relation $\succeq$ is arbitrage-sensitive. Let $x$ be any element of $C$. By arbitrage-sensitivity, for every $a \geq 0$, there exists $\mu=\mu(x, a)$ sufficiently large, such that $\mu x \succeq a m_{0}$. This implies that

$$
\mu x \in D(a),
$$

that is to say,

$$
x \in \frac{1}{\mu} D(a) .
$$

Consequently,

$$
x \in \bigcup_{\lambda>0} \lambda D(a)
$$

or, equivalently,

$$
x \in K(a)
$$

for every $a \geq 0$. Therefore,

$$
x \in \bigcap_{a \geq 0} K(a)
$$

and so $C \subseteq \bigcap_{a \geq 0} K(a)$.

Reciprocally, assume that $C \subseteq \bigcap_{a \geq 0} K(a)$. Let $x$ be any element of $C$. By hypothesis, $x \in \bigcap_{a \geq 0} K(a)$ and so, for every $a \geq 0$, there exists $\mu=\mu(x, a)$ large enough, such that $x \succeq \mu D(a)$. This implies that

$$
\frac{1}{\mu} x \in D(a)
$$

that is to say,

$$
\frac{1}{\mu} x \succeq a m_{0}
$$

and so the preference relation is arbitrage-sensitive.

Now, we need the following Definition [5].

Definition 5. The preference relation $\succeq$ is said to be downside-sensitive if, for every $x \notin C$, there is a desirability level $a \geq 0$ such that the ray generated by $x$ is dominated by $a m_{0}$, that is to say, am $\npreceq\{\lambda x\}_{\lambda>0}$.

We can enunciate the following statement.

Proposition 3. If the preference relation $\succeq$ is arbitrage-sensitive, then $C=\bigcap_{a \geq 0} K(a)$ if, and only if, $\succeq$ is downside-sensitive.

Proof. First, assume that the preference relation $\succeq$ is downside-sensitive. As $\succeq$ is arbitrage-sensitive, $C \subseteq \bigcap_{a \geq 0} K(a)$. Let us suppose that there exists a $x \in \bigcap_{a \geq 0} K(a)$ such that $x \notin C$. Then, for every $a \geq 0$, there exists $\mu=\mu(x, a)$ large enough, such that $x \succeq \mu a m_{0}$. Therefore, any multiple of $m_{0}$ dominates $x$, whereby downside-sensitivity does not hold.

Reciprocally, assume that $C=\bigcap_{a \geq 0} K(a)$ and consider an element $x \notin C$. As $x \notin \bigcap_{a \geq 0} K(a)$, then there exists an $a \geq 0$ such that, for every $\lambda>0, x \notin \lambda K(a)$. Consequently, the ray $\left\{\frac{1}{\lambda} x\right\}_{\lambda>0}$ does not intersect $K(a)$ and so $\frac{1}{\lambda} x \nsucceq a m_{0}$, for every $\lambda>0$, whereby $a m_{0}$ dominates the ray $\left\{\frac{1}{\lambda} x\right\}_{\lambda>0}$.

Finally, we are going to relate NA with no good deals (see [5]).

Theorem 3. If the preference relation $\succeq$ is arbitrage- and downside-sensitive, then NA holds if, and only if, for every $a \geq 0, K(a) \cap F=\varnothing$. 
Proof. First, assume that NA holds and suppose that there exists $a \geq 0$ such that $K(a) \cap F \neq \varnothing$. (The parameter $a$ can be supposed to be greater than zero since, otherwise, $K(0) \cap F=C \cap F \neq \varnothing$ and this is not possible by the hypothesis.) If $x \in K(a) \cap F$, then $p(x) \leq 0$ and $x \in K(a)$. Therefore, there exists $\lambda>0$ such that $x \in \lambda D(a)$, that is to say, $x=\lambda y$, where $y \succeq a m_{0}$. Thus, $x \succeq \lambda a m_{0}$ and then $p(x)=\operatorname{\lambda ap}\left(m_{0}\right)>0$, which is a contradiction.

Reciprocally, if $K(a) \cap F=\varnothing$, for every $a \geq 0$, obviously one has $\bigcap_{a \geq 0} K(a) \cap F=\varnothing$. By Proposition $3, \bigcap_{a \geq 0} K(a)=C$, whereby $C \cap F=\varnothing$ and then NA holds.

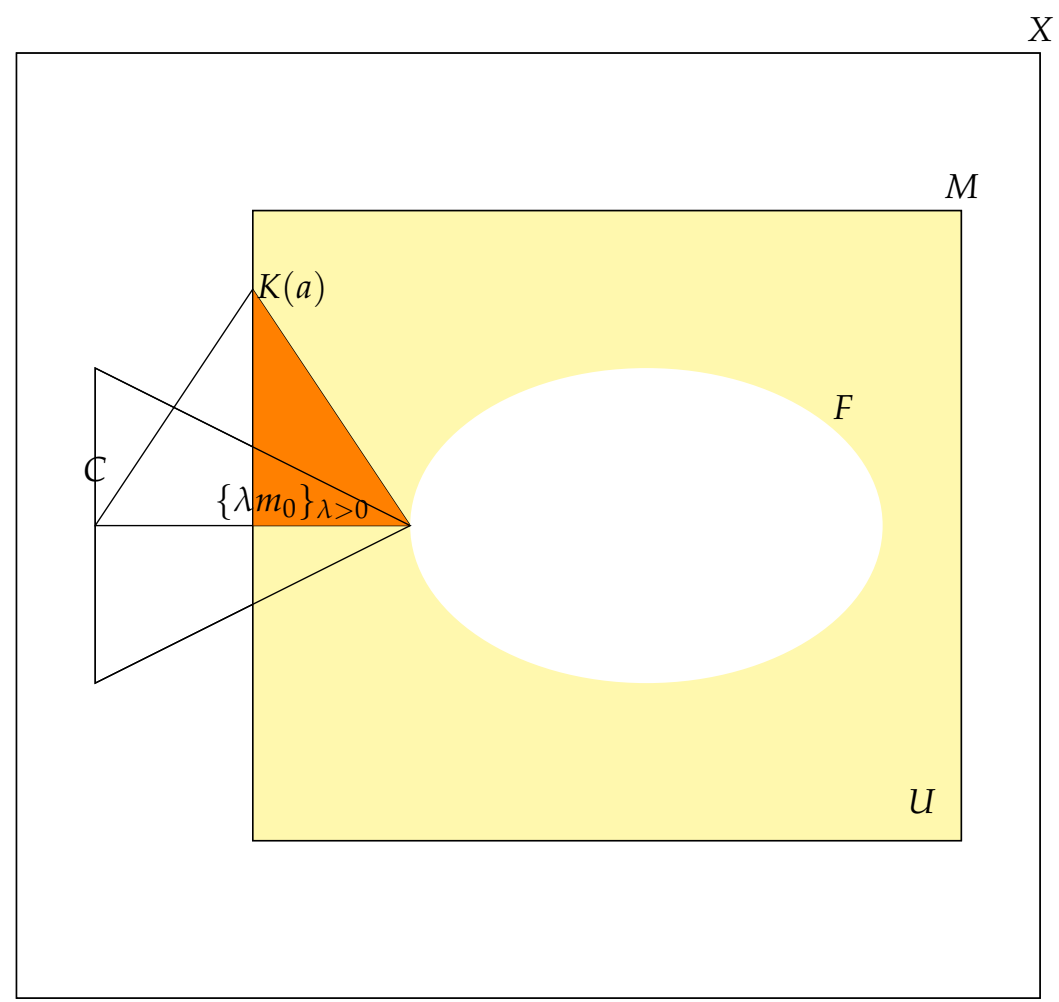

Figure 8. In yellow, elements in $M$ with a unique (positive) price. In orange, elements in $K(a) \cap M$ with a unique (positive) price. In white, elements in $F$ with a unique (negative or null) price. Source: Own elaboration.

The next statement provides a topological characterization where the cone is involved.

Theorem 4. If $p$ is a continuous map NA holds if, and only if, $C \subseteq \operatorname{Ext}(F)$, where Ext $(F)$ is the exterior of $F$ in the topological space $M$.

Proof. Let us suppose that $p$ is a continuous map. It is well known that $\left.\left.F=p^{-1}(]-\infty, 0\right]\right)$, ker $p=p^{-1}(\{0\})$ and that $\left.\left.\{0\}=b(]-\infty, 0\right]\right)$, being $b(A)$ the boundary of $A$. So, taking into account that in general $f^{-1}(b(A)) \supseteq b\left(f^{-1}(A)\right)$, then ker $f \supseteq b(F)$. Reciprocally, for every $x \in \operatorname{ker} f, p(x)=0$. Let us consider any neighborhood of $x, E(x)$. It can be stated that there exists a $x_{0} \in E^{*}(x)$ such that $p\left(x_{0}\right) \leq 0$, because, otherwise, if $p(z)$ is greater than 0 for all $z \in E^{*}(x)$, then, by the continuity of $p, p(x)$ would be also greater than 0 , which is a contradiction. Thus, $E^{*}(x) \cap F \neq \varnothing$ and $x \in b(F)$. Therefore, $\operatorname{ker} p=b(F)$.

\section{An Approach Including Time}

The approach proposed by [4] does not consider explicitly the variable "time". Nevertheless, the inclusion of time in the theory on securities, contingent claims, etc. is essential [20]. In effect, sometimes, the contingent claim is subject to the realization or not of a random event in the future so time becomes an inherent element to the concept of a contingent claim. 
In this section, we are going to consider a special class of contingent claims inside the linear space $X$. Some of them will belong to the marketed space $M$. In effect, denote by $[t, a]$ the right, arisen at $t$, to receive 1 euro after a period of length $a$, conditioned to the occurrence of an event, or the value of a random variable.

Let $S$ be the set of the previously defined contingent claims and consider the real vector space $W$ spanned by $S$ :

$$
W:=\operatorname{span}(S) .
$$

The contingent claim $[t, a]$ can be defined by the random variable $X_{t, a}$ which is defined as follows:

$$
X_{t, a}= \begin{cases}1, & \text { if } t+a \leq \tau_{A}(t) \\ 0, & \text { if } t+a>\tau_{A}(t)\end{cases}
$$

where $\tau_{A}(t)$ is the random variable, called the stopping time or first-hit time [21,22] associated to a measurable subset $A$ of the state space $S$ of a probability space $(\Omega, \sigma, P)$, defined by:

$$
\tau_{A}(t)(\omega):=\inf \left\{a \in \mathbb{R}^{+}: Y_{t, a}(\omega) \in A\right\},
$$

that is to say, the first time at which the certain random variable

$$
Y_{t, a}: \Omega \rightarrow S,
$$

reaches subset $A$. Said in other words, $\tau_{A}(t)(\omega)$ can be interpreted as the lapse of time until which a critical event $A$ occurs (called, in certain contexts, a "survival time").

The space $W$ is composed by the finite formal sums:

$$
\sum_{s \in S} a_{s} s
$$

Thus, if $s=[t, a]$, a product $x s=x[t, a]$ can be defined as the right, arisen at $t$, to receive $x$ euros after a period of length $a$, conditioned by the occurrence of an event or the value of a random variable. On the other hand, the sum $\sum_{s \in S} a_{s} s$ represents a portfolio of contingent claims with different amounts and expirations.

In this way, every price map $p$ will assign at least a real number to each one of the elements in $W$. It is well known [3] that the absence of arbitrage holds if and only if $p$ is a homomorphism of vector spaces (which implies, among others, that $p$ is a map).

Observe that the vectors

$$
[t, a+b]
$$

and

$$
p([t+a, b])[t, a]
$$

lead to the same conditioned right (to receive 1 euro at instant $t+a+b)$. (Observe that $p([t+a, b])[t, a]$ is the right to receive $p([t+a, b])$ euros at time $t+a$, but with this money one can buy the right to receive 1 euro at time $t+a+b$.) Thus, they can be considered as two different representations of the same vector. Thus, the law of one price applies which, as shown, "is less restrictive than the absence of arbitrage because it deals only with the case in which two assets are identical but have different prices" [1]. So,

$$
p([t, a+b])=p(p([t+a, b])[t, a]),
$$

which implies that (Figure 9):

$$
F(t, a+b)=F(t+a, b) F(t, a),
$$


where we have simplified the notation, by writing:

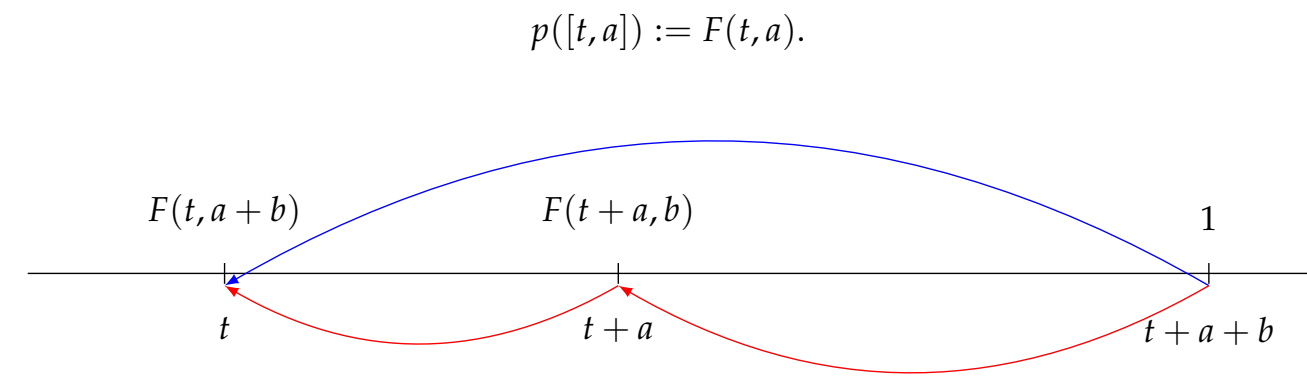

Figure 9. Additivity of the discount function $F(t, a)$. Source: Own elaboration.

From the last equation, it would remain:

$$
F(t+a, b)=\frac{F(t, a+b)}{F(t, a)}
$$

that is to say, the spot price at time $t+a$ of a bond promising 1 euro at $[t+a, t+a+b]$ (i.e., $F(t+a, b)$ ) coincides with its forward price (i.e., $\left.\frac{F(t, a+b)}{F(t, a)}\right)$.

Specifically, if a contract $[t, a], a \geq 0$, pays surely 1 euro at time $t+a$ (that is, the dependence on the occurrence of any event is removed) and $F(t+a, b)$ is a forward price, to be paid at time $t+a$ to receive 1 euro at time $t+a+b$ for sure, then $F(t, a+b)=F(t, a) F(t+a, b)$ is a well-known equation which can be proved through simple arbitrage arguments.

The fact of $p$ being a map means that, although a same right can be written using different expressions, its price is unique. That is to say, different strategies cannot lead to different investors paying different prices for the same right.

Example 3. The right to receive 1000 euros within three years (instant 3) costs today (instant 0) 847.46 euros, if the hyperbolic discounting is used at $6 \%$ per year. The same right costs 892.86 euros at instant 1 and 943.40 euros at instant 2. Thus, the right to receive 1000 euros within three years coincides with the right to receive 892.86 within one year (which implies receiving 1000 euros within two years) and coincides with the right to receive 943.40 euros within two years (which, at the same time, allows us to receive 1000 euros within one year), that is to say:

$$
1000[0,3]=892.86[0,1]=943.40[0,2] .
$$

Nevertheless, the price of this right is going to depend on the concrete used linear combination, being the price of each one $847.46,842.32$ and 842.32 euros, respectively.

Next, we will try to describe the topology induced in the space of the contingent claims previously defined. To do this, we will describe the neighborhoods of an arbitrary point in this topological space, giving an intuitive interpretation from a financial point of view. In effect, consider an arbitrary contingent claim $x[t, a]$. For each one of the possible values of $x$, there exists a neighborhood which represents not the indifference among its elements but a concept of proximity (Figure 10). 


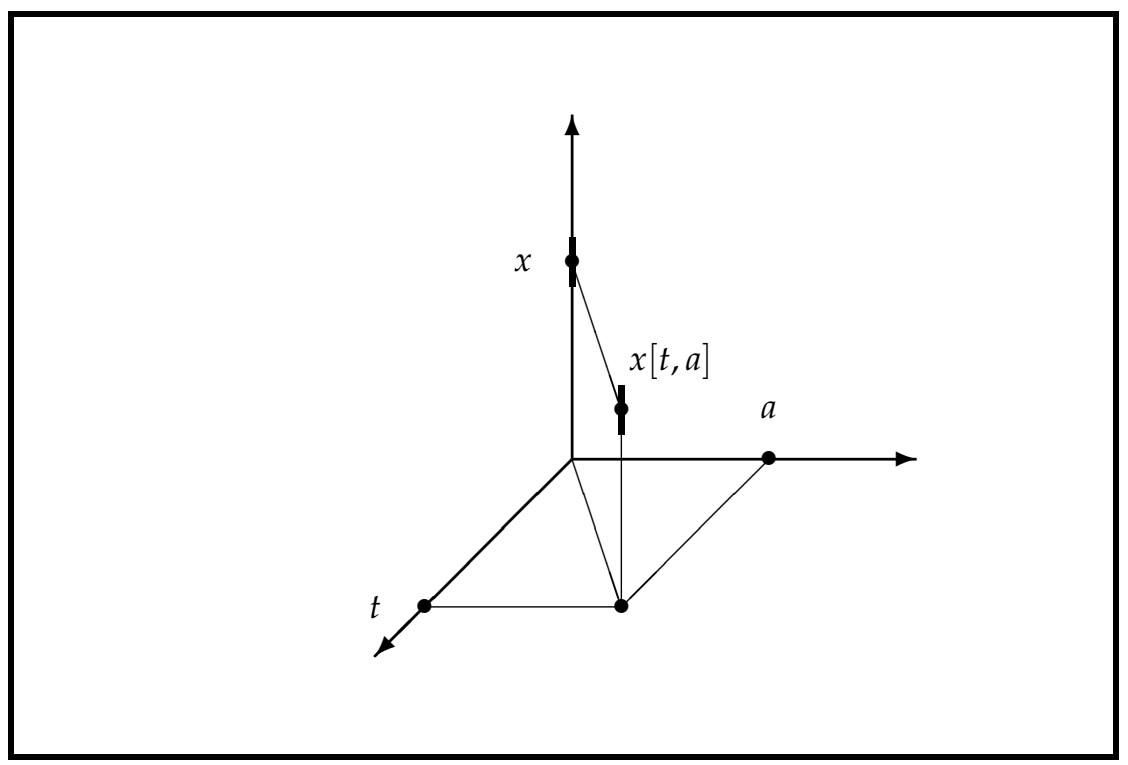

Figure 10. Neighborhood of a point in the topological space. Source: Own elaboration.

\section{Discussion}

This paper has dealt with the problem of pricing contingent claims $[23,24]$ by deducing some algebraic conditions under which there exists a single price for a specific contingent claim which will not permit arbitrage profits.

In this context, the paper by Clark [3] is an abstraction of the work by Kreps [7] which, in turn, is a generalization of the seminal paper by Harrison and Kreps [6]. In effect, this last paper considers a two-dated $(0$ and $T)$ economy with uncertainty and consumption bundles of the form $(c, X) \in \mathbb{R} \times X$, where $X$ is a space of random variables on $(\Omega, \mathcal{F}, P)$. The couple $(c, X)$ represents $c$ units of consumption at time 0 and $X(\omega)$ units of consumption if the state at time $T$ is $\omega$.

Later, Kreps [7] generalizes the framework of this foundational model to a general topological space endowed with a cone and a set of feasible contingent claims (described by the former random variables). However, Clark [3,4] weakens the restrictions of the former model by allowing that the price is a linear correspondence and not a functional, by demonstrating that NA holds if and only if the price linear map $p$ is strictly positive [4].

Our paper proposes a wider setting where the conditions required to the model have been relaxed at maximum by using a cone that is not necessarily convex, which gives rise to an ordering only satisfying reflexivity. It is well known that the Fundamental Theorem of Asset Pricing states the equivalence of "absence of arbitrage, existence of a positive linear pricing rule, and the existence of an optimal demand for some agent who prefers more to less" [1].

Observe that, whilst the papers by Clark [3,4] are focused on the existence of a positive linear pricing rule, our paper partially follows the line of Kreps [7] by showing the existence of an optimal demand. In effect, Kreps introduced the concept of a viable price system $(M, \pi)$ for which there exist some $\succeq$ and $\left(r^{*}, m^{*}\right) \in \mathbb{R} \times M$ such that $r^{*}+\pi\left(m^{*}\right) \leq 0$ and $\left(r^{*}, m^{*}\right) \succeq(r, m)$ for every $(r, m) \in \mathbb{R} \times M$ such that $r+\pi(m) \leq 0$. In our paper, this condition is represented by the statement (iv) in Theorem 1 .

Finally, observe that the optimal demand is linked to an ordering $\succeq$ which, in turn, is associated to a cone. The treatment of all possible cones (not necessarily including the government bond) have been analyzed in this paper and, more specifically, the relationship between NA and no good-deals condition [5] has been analyzed in Theorem 3 .

Other types of simple arbitrage and no arbitrage (NA) opportunities can be defined in this context, and it is of interest to analyze possible implications between them. In effect, the initial definition of NA can be completed by requiring some additional and logical conditions, giving rise to the topological concepts of: 
- $\quad$ no approximate arbitrage (NAA) [3], and

- $\quad$ no free lunches (NFL) [7].

On the other hand, in the Introduction a discrete-time structure was introduced and the proposed approach also allows embedding continuous-time models. In effect, a continuous-time setting could be considered where $t \in[0, T]$. A filtered probability space $\left(\Omega, \mathcal{F},\left(\mathcal{F}_{t}\right)_{0 \leq t \leq T}, P\right)$ is given, where $\Omega$ is the state space, $\mathcal{F}$ is a $\sigma$-algebra representing the set of events, $\left(\mathcal{F}_{t}\right)_{0 \leq t \leq T}$ is a filtration, and $P$ is a probability measure. Additionally, $\mathcal{F}_{0}$ includes the null sets of $\mathcal{F}$ and right continuity implies that, given a random variable $\tau: \Omega \rightarrow \mathbb{R}$, for every $t$, it can be stated that $\{\omega: \tau(\omega) \leq t\} \in \mathcal{F}_{t}$ if, and only if, $\{\omega: \tau(\omega)<t\} \in \mathcal{F}_{t}$. Finally, $\mathcal{F}_{T}=\mathcal{F}[15]$.

In any of the two proposed models (discrete-time or continuous-time), we can highlight the probabilistic concepts of:

- $\quad$ no unbounded profits with bounded risk (NUPBR) [25],

- no arbitrage of the first kind (NA1) [26],

- $\quad$ no free lunch with vanishing risk (NFLVR) [15,27], and

- $\quad$ no free lunch with bounded risk (NFLBR) [28].

In this way, most existing literature is devoted to obtaining some necessary and sufficient conditions for the existence of NUPBR and NFLVR. It is indicated in $[29,30]$ that, apart from NFL, NUPBR, NA1, NFLBR and NFLVR, many alternative NA definitions have arisen during the last two decades: no increasing profit (NIP) and no strong arbitrage (NSA).

A related approach has been adopted in [31,32], modelling portfolio values rather than trading strategies. In effect, the topological approach pursued in this paper abstracts from an explicit description of trading strategies. In this way, we can see a connection with the framework presented by Kabanov (1997) which does not assume discrete-time or continuous-time, nor any model for the price processes. Instead, Kabanov introduces a more general setting "with value processes as the primary objects" [32] and is based on a topological linear space and a convex cone. Specifically, let $\mathcal{S}$ be the linear space of semimartingales $X$ defined on a finite interval $[0, T]$ and starting from $0 . \mathcal{S}$ is a Fréchet space with the quasinorm:

$$
\mathbf{D}(X):=\sup _{h \in \mathcal{U}_{1}} E\left(1 \wedge\left|h \cdot X_{T}\right|\right),
$$

where $\mathcal{U}_{1}$ is the set of all predictable processes $h$ taking values in the interval $[-1,+1]$.

Let $\chi^{1}$ a closed subset (take into account that $\mathbf{D}$ induces the so-called Émery topology) of process $X \geq-1$, including 0 , which satisfies the following condition: For every $X, Y \in \chi^{1}$ and every non-negative bounded predictable processes $H$ and $G$ with $H G=0, Z=H \cdot X+G \cdot Y \geq-1$ implies $Z \in \chi^{1}$. Let $\chi$ be the cone generated by $\chi^{1}$, that is to say,

$$
\chi:=\cup_{\lambda>0} \lambda \chi^{1}
$$

$\chi$ can be interpreted as the set of value processes. Kabanov proposed a one-step model of frictionless market admissible strategies such that the convex cone

$$
R_{T}^{0}:=\left\{X_{T}: X \in \chi\right\}
$$

(the set of final portfolio values corresponding to zero initial endowment) contains only (scalar) random variables bounded from below.

In the general semimartingale model, one has the following chain of implications $[28,30]$ :

$$
\mathrm{NFL} \Rightarrow \mathrm{NFLBR} \Rightarrow \mathrm{NFLVR} \Rightarrow \mathrm{NUPBR} \Leftrightarrow \mathrm{NA} 1 \Rightarrow \mathrm{NSA} \Rightarrow \mathrm{NIP} .
$$

Finally, in this section, it is very important to highlight the economic and financial implications of the different NA conditions. According to [30], NA condition is related to the concept of market viability, "in the sense of solvability of the portfolio optimization problem for some hypothetical agent who prefers more to less", apart from to solve the fundamental problems of pricing and hedging. 
In this way, it is obvious that NIP is not a sufficient condition and that NSA is a necessary condition to ensure market viability. Of course, the most interesting thing is to find which NA condition is equivalent to market viability. In this way, NA1 holds [30] if, and only if, there exists an optimal portfolio for an agent with regular preferences. Moreover, it is stated that NA1 (strictly weaker than the classical NFLVR) is a minimal condition in order to ensure a noteworthy form of market viability.

Following [1], the "absence of arbitrage is more general than equilibrium because it does not require all agents to be rational". Additionally, [30] points out that "the existence of arbitrage profits is incompatible with the possibility of a competitive equilibrium". Finally, [33] state that "any market equilibrium must be consistent with no arbitrage profits".

It is well known that the efficiency of a market can be seen from three perspectives [34,35]. Any form of efficiency aims to avoid the existence of a predictable pattern which could give rise to a profitable trading strategy [36]. More specifically, the weak-form efficiency refers to the information exhibited by assets in their past prices which could eventually envision the future value of these assets. The semi-strong efficiency refers to current prices which reflect all information available to the public. Finally, the strong-form efficiency reflects the context in which all public and private information is included in the quote of assets.

Definitively, in case of the efficient market hypotheses, no abnormal profits could be obtained by investors, and profitable investing strategies with minimum risk (viz speculation) are not applicable.

\section{Conclusions}

This paper presents a novel approach to the Arbitrage Theory introduced by Clark $[3,4]$ by providing some new necessary and sufficient conditions to characterize the absence of arbitrage and by using purely algebraic tools and structures. Traditionally, the different kinds of absence of arbitrage have been analyzed from a topological or a probabilistic point of view. Consequently, this paper fills the gap between both mathematical disciplines by only including algebraic concepts.

As seen in Section 6, the model described in Section 2 is wide enough to include most financial markets. In effect, we have used the vector space of all contingent claims with an ordering (a binary relation satisfying only reflexivity) and a cone (not necessarily convex) which reflects the direction of increase of the economic value. In a beginning, there is a price correspondence over the so-called subspace of marketed claims. Clark [3] showed that a necessary and sufficient condition for NA is that the price correspondence is a homomorphism of vector spaces.

However, in this paper we have divided the algebraic characterizations of NA into two groups: those concerning the preference relation defined on the space of contingent claims (Section 3) and those involving the cone (Section 4). In this last section, following the work by Černý and Hodges [5], we have introduced new cones which do not necessarily include government bonds. Subsequently, the concepts of arbitrage- and downside-sensitivity have been incorporated to analyze the relationship between the absence of arbitrage and no good deals.

The framework of this paper is an efficient market involving the impossibility of profitable risk-zero arbitrage which necessarily implies that the preference relation associated with the bond price is consistent [37]. In this case, the price at time $t$ of a discount bond promising 1 euro at maturity $t+a+b$ is the same as the price at time $t$ of a discount bond promising $F(t+a, b)$ at maturity $t$. This property gives rise to the so-called additive discount functions which satisfy the following functional equation:

$$
F(t, a+b)=F(t, a) F(t+a, b),
$$

for every $t$ and $a, b \geq 0$. This means that the agent does not obtain any advantage when he divides the complete period of time, $[t, t+a+b]$, of the investment into two intervals, $[t, t+a]$ and $[t+a, t+a+b]$. (In [38] these discount functions were labeled decomposable financial laws.) The algebraic characterizations of this property have been studied from several points of view [39] and, particularly, by considering the Algebraic Automata Theory [40]. 
Funding: The author gratefully acknowledges financial support from the Spanish Ministry of Economy and Competitiveness [National R\&D Project "La sostenibilidad del Sistema Nacional de Salud: reformas, estrategias y propuestas", reference: DER2016-76053-R].

Acknowledgments: I am very grateful for the valuable comments and suggestions offered by two anonymous referees and the participants in the congress "Rings, modules, and Hopf algebras. A conference on the occasion of Blas Torrecillas' 60th birthday", held in Almería (Spain), May 13-17, 2019.

Conflicts of Interest: The author declares no conflict of interest.

\section{Abbreviations}

The following abbreviations are used in this manuscript:

$\begin{array}{ll}\text { NA } & \text { No Arbitrage } \\ \text { NAA } & \text { No Approximate Arbitrage } \\ \text { NFL } & \text { No Free Lunches } \\ \text { NUPBR } & \text { No Unbounded Profits with Bounded Risk } \\ \text { NA1 } & \text { No Arbitrage of the First Kind } \\ \text { NFLVR } & \text { No Free Lunch with Vanishing Risk } \\ \text { NFLBR } & \text { No Free Lunch with Bounded Risk } \\ \text { NIP } & \text { No Increasing Profit } \\ \text { NSA } & \text { No Strong Arbitrage } \\ \text { USD } & \text { U.S. dollar } \\ \text { EUR } & \text { Euro } \\ \text { GBP } & \text { British pound sterling }\end{array}$

\section{References}

1. Dybvig, P.H.; Ross, S.A. Arbitrage. In The New Palgrave Dictionary of Economics; Palgrave Macmillan: London, UK, 2008; pp. 1-12.

2. Cruz Rambaud, S. Arbitrage theory with state-price deflators. Stoch. Models 2013, 29, 306-327. [CrossRef]

3. Clark, S.A. The valuation problem in arbitrage price theory. J. Math. Econ. 1993, 22, 463-478. [CrossRef]

4. Clark, S.A. Arbitrage approximation theory. J. Math. Econ. 2000, 33, 167-181. [CrossRef]

5. Černý, A.; Hodges, S. The theory of good-deal pricing in financial markets. In Mathematical Finance. Bachelier Congress 2000; Geman, H., Madan, D., Pliska, S.R., Vorst, T., Eds.; Springer: Berlin, Germany, 2000.

6. Harrison, M.J.; Kreps, D.M. Martingales and arbitrage in multiperiod securities markets. J. Econ. Theory 1979, 20, 381-408. [CrossRef]

7. Kreps, D.M. Arbitrage and equilibrium in economies with infinitely many commodities. J. Math. Econ. 1981, 8, 15-35. [CrossRef]

8. Steiner, M.; Schneider, S. Time varying risk premia. In Decision Technologies for Computational Finance; Springer Science + Business Media: Dordrecht, Germany, 1998; pp. 25-48.

9. LeRoy, S.F.; Werner, J. Principles of Financial Economics; Cambridge University Press: Cambridge, UK, 2014.

10. Dugundgi, J. Topology; Allyn and Bacon: Boston, MA, USA, 1967.

11. Clark, S.A. An infinite-dimensional LP duality theorem. Math. Oper. Res. 2003, 28, 233-245. [CrossRef]

12. Werner, J. Arbitrage and the existence of competitive equilibrium. Econometrica 1987, 55, $1403-1418$. [CrossRef]

13. Díez de Castro, L.; Mascareñas, J. Ingeniería Financiera. La Gestión de los Mercados Financieros Internacionales, 2nd ed.; McGraw-Hill: Madrid, Spain, 1994.

14. Privault, N. Notes on Stochastic Finance; 2019. Available online: Http://www.ntu.edu.sg/home/nprivault/ indext.html (accessed on 22 May 2019).

15. Jarrow, R.A. Continuous-Time Asset Pricing Theory. A Martingale-Based Approach; Springer: New York, NY, USA, 2018.

16. Ross, S.A. Return, risk and arbitrage. In Risk and Return in Finance; Friend, I., Bicksler, J., Eds.; Ballinger: Cambridge, MA, USA, 1976.

17. Ross, S.A. The arbitrage theory of capital asset pricing. J. Econ. Theory 1976b, 13, 341-360. [CrossRef]

18. Ross, S. A simple approach to the valuation of risky streams. J. Bus. 1978, 51, 453-485 [CrossRef] 
19. Hausner, M.; Wendel, J.G. Ordered vector spaces. Proc. Am. Math. Soc. 1952, 3, 977-982. [CrossRef]

20. Debreu, G. Theory of Value; Wiley: New York, NY, USA, 1959.

21. Billingsley, P. Probability and Measure; Wiley: New York, NY, USA, 1995.

22. Resnick, S.I. A Probability Path; Birkhäuser: Boston, MA, USA, 1999.

23. Focardi, S.M.; Fabozzi, F. The Mathematics of Financial Modeling and Investment Management; John Wiley \& Sons, Inc.: Hoboken, NJ, USA, 2004.

24. Safra, Z. Contingent commodities. In The New Palgrave Dictionary of Economics; Palgrave Macmillan: London, UK, 2008; pp. 1-2.

25. Karatzas, I.; Kardaras, C. The numeraire portfolio in semimartingale financial models. Financ. Stoch. 2007, 11, 447-493. [CrossRef]

26. Kardaras, C. Market viability via absence of arbitrage of the first kind. Financ. Stoch. 2012, 16, 651-667. [CrossRef]

27. Delbaen, F.; Schachermayer, W. A general vision of the fundamental theorem of asset pricing. Math. Ann. 1994, 312, 215-250. [CrossRef]

28. Delbaen, F.; Schachermayer, W. Arbitrage and free lunch with bounded risk for unbounded continuous processes. Math. Financ. 1994, 4, 343-348. [CrossRef]

29. Hulley, H. Strict Local Martingales in Continuous Financial Market Models. PhD Thesis, University of Sydney, Sydney, Australia, 2009.

30. Fontana, C. Weak and strong no-arbitrage conditions for continuous financial markets. Int. J. Theor. Appl. Financ. 2015, 18, 1550005. [CrossRef]

31. Kabanov, Y.M. On the FTAP of Kreps-Delbaen-Schachermayer. In Statistics and Control of Stochastic Processes: The Liptser Festschrift; Kabanov, Y., Rozovskii, B.L., Shiryaev, A.N., Eds.; World Scientific: Singapore, 1997; pp. 191-203.

32. Kabanov, Y.M. Arbitrage theory. In Handbooks in Mathematical Finance. Option Pricing, Interest Rates and Risk Management; Jouini, E., Cvitanić, J., Musiela, M., Eds.; Cambridge University Press: Cambridge, UK, 2001; pp. 3-42.

33. Roll, R.; Ross, S.A. An empirical investigation of the arbitrage pricing theory. J. Financ. 1980, 35, $1073-1103$. [CrossRef]

34. Fama, E.F. Efficient capital markets: II. J. Financ. 1991, 46, 1575-1617. [CrossRef]

35. Fama, E.F. Market efficiency, long-term returns, and behavioral finance. J. Financ. Econ. 1998, 49, $283-306$. [CrossRef]

36. Kyriazis, N.A. A survey on efficiency and profitable trading opportunities in cryptocurrency markets. J. Risk Financ. Manag. 2019, 12, 67. [CrossRef]

37. Harvey, C.M. The reasonableness of non-constant discounting. J. Public Econ. 1994, 53, 31-51. [CrossRef]

38. Cruz Rambaud, S.; Ventre, A.G.S. Decomposable financial laws and profitability. Int. J. Uncertain. Fuzziness Knowl. Based Syst. 1998, 6, 329-344. [CrossRef]

39. Cruz Rambaud, S. Some new ideas in the concept of financial law. Riv. Mat. Sci. Econ. Soc. 1997, 1, $23-43$. [CrossRef]

40. Cruz Rambaud, S.; García Pérez, J. Financial laws as algebraic automata. Int. J. Intell. Syst. 2001, 16, 1085-1105. [CrossRef]

(C) 2019 by the author. Licensee MDPI, Basel, Switzerland. This article is an open access article distributed under the terms and conditions of the Creative Commons Attribution (CC BY) license (http://creativecommons.org/licenses/by/4.0/). 